\title{
MEASUREMENTS OF CYCLOTRON FEATURES AND PULSE PERIODS IN THE HIGH-MASS X-RAY BINARIES 4U 1538-522 AND 4U 1907+09 WITH THE INTERNATIONAL GAMMA-RAY ASTROPHYSICS LABORATORY
}

\author{
Paul B. Hemphill ${ }^{1}$, Richard E. Rothschild ${ }^{1}$, Isabel Caballero ${ }^{2}$, Katja Pottschmidt $^{3,4}$, Matthias Kühnel $^{5}$, \\ FELIX FÜRST $^{6}$, AND JÖRN WILMS ${ }^{5}$ \\ ${ }^{1}$ Center for Astrophysics and Space Sciences, University of California, San Diego, 9500 Gilman Drive, La Jolla, CA 920093-0424, USA; \\ pbhemphill@ physics.ucsd.edu \\ ${ }^{2}$ CEA Saclay, DSM/IRFU/SAp-UMR AIM (7158) CNRS/CEA/Université Paris 7, Diderot, F-91191 Gif-sur-Yvette, France \\ ${ }^{3}$ Center for Space Science and Technology, University of Maryland Baltimore County, 1000 Hilltop Circle, Baltimore, MD 21250, USA \\ ${ }^{4}$ CRESST and NASA Goddard Space Flight Center, Astrophysics Science Division, Code 661, Greenbelt, MD 20771, USA \\ ${ }^{5}$ Dr. Karl Remeis-Sternwarte and Erlangen Center for Astroparticle Physics, Sternwartstr. 7, D-96049 Bamberg, Germany \\ ${ }^{6}$ Cahill Center for Astronomy and Astrophysics, California Institute of Technology, MC 290-17, 1200 East California Boulevard, \\ Pasadena, CA 91125, USA \\ Received 2013 January 21; accepted 2013 August 30; published 2013 October 17
}

\begin{abstract}
We present a spectral and timing analysis of International Gamma-Ray Astrophysics Laboratory (INTEGRAL) observations of two high-mass X-ray binaries, 4U 1538-522 and 4U 1907+09. Our timing measurements for 4U 1538-522 find the pulse period to have exhibited a spin-up trend until approximately 2009, after which there is evidence for a torque reversal, with the source beginning to spin down to the most recently measured period of $525.407 \pm 0.001 \mathrm{~s}$. The most recent INTEGRAL observations of 4U 1907+09 are not found to yield statistically significant pulse periods due to the significantly lower flux from the source compared with 4U 1538-522. A spectral model consisting of a power-law continuum with an exponential cutoff and modified by two cyclotron resonance scattering features is found to fit both sources well, with the cyclotron scattering features detected at $\sim 22$ and $\sim 49 \mathrm{keV}$ for $4 \mathrm{U} 1538-522$ and at $\sim 18$ and $\sim 36 \mathrm{keV}$ for $4 \mathrm{U} 1907+09$. The spectral parameters of $4 \mathrm{U}$ 1538-522 are generally not found to vary significantly with flux and there is little to no variation across the torque reversal. Examining our results in conjunction with previous work, we find no evidence for a correlation between cyclotron line energy and luminosity for 4U 1538-522. 4U 1907+09 shows evidence for a positive correlation between cyclotron line energy and luminosity, which would make it the fourth, and lowest luminosity, cyclotron line source to exhibit this relationship.
\end{abstract}

Key words: pulsars: individual (4U 1538-522, 4U 1907+09) - stars: magnetic field - stars: oscillations X-rays: binaries - X-rays: stars

Online-only material: color figures

\section{INTRODUCTION}

High-mass X-ray binaries (HMXBs) are a class of binary systems consisting of a neutron star with a high-mass $\left(\sim 10-20 M_{\odot}\right)$, main-sequence companion. X-ray emission from the neutron star is driven primarily by accretion of material from the main-sequence companion, typically via either Roche lobe overflow in the case of less-massive companions or the stellar wind of a higher mass companion. The neutron star's strong $\left(\sim 10^{12-13} \mathrm{G}\right)$ magnetic field channels accrete material onto the magnetic poles, forming an accretion column where the majority of the X-ray luminosity is produced via inverse Compton scattering of thermal photons produced at the stellar surface and bremsstrahlung radiation from within the column. Obtaining a detailed physical picture of this process is still an open problem in X-ray astrophysics. Early work (e.g., Meszaros \& Nagel 1985; Klein et al. 1996) was generally limited in its ability to properly reproduce the accreting neutron star spectrum, due to the extreme complexity of the problem. Recently, however, work by Becker \& Wolff (2007) has produced promising results, successfully reproducing the continuum spectra of several high-luminosity X-ray pulsars. In addition to the base continuum, several dozen HMXBs display absorption-like features in their spectra that are identified as cyclotron resonance scattering features (CRSFs, also known as cyclotron lines), produced by the resonant scattering of photons on electrons moving in the magnetic field; the physical modeling of these features has also seen significant progress in work by Schönherr et al. (2007). Work is currently underway to merge the continuum and cyclotron line models into a single model capable of producing an accurate, physically motivated model for accreting neutron stars (Schwarm et al. 2013; Schwarm 2013).

The accretion-powered X-ray pulsar 4U 1538-522 was discovered in the early 1970 s by the Uhuru satellite (Giacconi et al. 1974). Its main-sequence companion is the $\sim 20 M_{\odot} \mathrm{B} 0$ star QV Nor (Reynolds et al. 1992). X-ray pulsations were first observed from the vicinity of 4U 1538-522 by Becker et al. (1977) and Davison (1977); Davison et al. (1977) identified these as coming from $4 \mathrm{U} 1538-522$. The distance to $4 \mathrm{U} 1538-522$ has been variously estimated to be $5.5 \pm 1.5$ (Crampton et al. 1978), $6.0 \pm 0.5$ (Ilovaisky et al. 1979), $6.4 \pm 1.0$ (Reynolds et al. 1992), and 4.5 (Clark 2004) kpc. Infrequent observations postdiscovery showed $4 \mathrm{U}$ 1538-522 to be spinning down until the late 1980s, reaching a maximum period of $\sim 531 \mathrm{~s}$ (Makishima et al. 1987; Davison 1977; Becker et al. 1977), after which extended BATSE monitoring between 1990 and 1995 by Rubin et al. (1997) showed the source to have transitioned to a longterm spin-up trend that would last until approximately 2009, when monitoring by the Gamma-ray Burst Monitor (GBM) on board the Fermi satellite, along with this work, show the 
source to have transitioned to a spin-down state (see Figure 2). The early OSO-8 and Ariel-5 observations by Becker et al. (1977) and Davison et al. (1977) also established a 3.7 day orbital period and provided the first picture of the binary orbit of the system, with the orbital parameters being updated by Makishima et al. (1987), Clark (2000), and Mukherjee et al. (2006). The exact characteristics of 4U 1538-522's orbit remain somewhat uncertain: the three aforementioned references find three different values for the eccentricity, with Makishima et al. adopting $e=0.08 \pm 0.05$, Clark finding solutions for both circular and elliptical $(e=0.174 \pm 0.015)$ orbits, and Mukherjee et al. supporting Clark's elliptical solution. Efforts to determine the mass of 4U $1538-522$ by van Kerkwijk et al. (1995) adopted a circular orbit, while recent work by Rawls et al. (2011) finds a mass of $0.87 \pm 0.07 M_{\odot}$ for Clark's elliptical orbit and $1.104 \pm 0.177 M_{\odot}$ for the circular solution, making $4 \mathrm{U}$ 1538-522 potentially one of the lightest neutron stars known. The spectrum of $4 \mathrm{U} 1538-522$ is fit well by a power law with an exponential cutoff (Makishima et al. 1987), with CRSFs at 22 (Clark et al. 1990) and $\sim 47 \mathrm{keV}$, tentatively reported in BeppoSAX data by Robba et al. (2001), but not confirmed until a combined RXTE and International Gamma-Ray Astrophysics Laboratory (INTEGRAL) analysis by Rodes-Roca et al. (2009).

$4 \mathrm{U} 1907+09$ is another wind-accreting X-ray binary, also discovered by Uhuru (Giacconi et al. 1971). It is similar to $4 \mathrm{U} 1538-522$ in its long pulse period $(\sim 440 \mathrm{~s})$, short orbital period ( $~ 8.4$ days), and optical companion $(08 / 09$, as found by Cox et al. 2005). For $\sim 15$ yr following the identification of the source as a pulsar by Makishima et al. (1984), the pulse period increased steadily from $437.4 \mathrm{~s}$ in 1984 (Makishima et al. 1984) to $\sim 441 \mathrm{~s}$ in 1998 , when the spin-down trend began to slow, leading to a torque reversal in 2005 (Fritz et al. 2006). This was followed relatively quickly by another reversal in mid2007 (Inam et al. 2009; Şahiner et al. 2011) and the source began following approximately the same spin-down trend as it had between 1987 and 1998, a trend that thus far shows no sign of changing. The initial determination of the $\sim 8.4$ day orbital period showed flaring twice per orbit, suggesting the source was in orbit around a Be-type star (Marshall \& Ricketts 1980), although subsequent optical observations indicated that the companion was more likely a supergiant (van Kerkwijk et al. 1989). Recent optical (Cox et al. 2005) and infrared (Nespoli et al. 2008) observations have more firmly established the companion as a O8/O9 supergiant, with Cox et al. finding a distance of $5.0 \mathrm{kpc}$ to the source, while Kostka \& Leahy (2010) have shown that the most likely scenario to produce the observed flaring involves a dense stellar wind along with a trailing stream of material between the neutron star and companion. Observations with Ginga established the existence of an absorption feature, identified as a CRSF, at $\sim 19 \mathrm{keV}$ (Mihara 1995; Makishima et al. 1999), with a second at $\sim 39 \mathrm{keV}$ detected in BeppoSAX data by Cusumano et al. (1998).

In this paper, we first present a summary of the observations used in our analysis in Section 2, followed by a brief discussion of the pulse timing measurements made for each source in Section 3. Section 4 presents the spectral analysis of each source, where the large energy range covered by the combination of the INTEGRAL Soft Gamma-Ray Imager (ISGRI) and the Joint European X-ray Monitor (JEM-X) instruments proves advantageous. Finally, we discuss these results in the context of recent theoretical results regarding accretion onto magnetic poles in Section 5.
Table 1

Sources Included in the Background Subtraction Routine for 4U 1538-522 and 4 U 1907+09, with 20-40 keV ISGRI Counting Rates

\begin{tabular}{lccc}
\hline \hline Source & Counts s $^{-1 \text { a }}$ & Source & Counts s $^{-1}$ \\
\hline 4U 1538-522 & 3.06 & 4U 1907+09 & 1.42 \\
\hline 4U 1700-377 & 32.4 & GRS 1915+105 & 44.9 \\
GX 339-4 & 22.2 & 4U 1909+07 & 2.26 \\
OAO 1657-415 & 11.3 & 4U 1916-053 & 1.76 \\
4U 1626-67 & 6.70 & XTE J1855-026 & 1.30 \\
IGR J16320-4751 & 5.03 & Ser X-1 & 1.24 \\
GX 340+0 & 4.78 & SS 433 & 1.23 \\
H 1608-522 & 4.25 & IGR J19140+0951 & 1.12 \\
H 1636-536 & 3.17 & IGR J18483-0311 & 0.92 \\
IGR J16318-4848 & 3.16 & Aq1 X-1 & 0.79 \\
H 1705-440 & 2.23 & Ginga 1843+009 & 0.69 \\
PSR B1509-58 & 1.42 & XTE J1901+014 & 0.39 \\
4U 1630-47 & ND $^{\mathrm{b}}$ & SWIFT J1753.5-0127 & ND \\
XTE J1652-453 & $\mathrm{ND}^{\mathrm{b}}$ & 4U 1812-12 & ND \\
$\quad \ldots$ & $\ldots$ & GX 17+2 & ND \\
$\ldots$ & $\ldots$ & PSR J1846-0258 & ND \\
$\ldots$ & $\ldots$ & 3A 1850-087 & ND \\
\hline
\end{tabular}

Notes.

a $20-40 \mathrm{keV}$ ISGRI count rates.

b ND: not detected in the mosaic image.

\section{OBSERVATIONS}

We used two of the instruments on board INTEGRAL: the ISGRI (Lebrun et al. 2003), which is the upper layer of the Imager on Board the INTEGRAL Satellite (Ubertini et al. 2003), a coded-mask telescope with a $9^{\circ} \times 9^{\circ}$ fully coded field of view (FOV), and JEM-X 1 and 2 (Lund et al. 2003), twin codedmask telescopes with circular FOVs of diameter 4.8. Early in the INTEGRAL mission, only one JEM-X telescope was in operation at any given time, out of concern for the lifetime of the microstrip anodes used in the detectors, but a lowered operating voltage alleviated these concerns and recent (post-2009) observations have both detectors running simultaneously. The INTEGRAL satellite has a $\sim 3$ day orbit; the science data for each orbit (or "revolution") are divided into $\sim 2 \mathrm{ks}$ long pointings called Science Windows (SCWs). The nominal energy range of ISGRI is $15 \mathrm{keV}-1 \mathrm{MeV}$, but we follow the ISGRI team's recommendation of a lower bound of $18 \mathrm{keV}$ for data taken prior to revolution 848 and $20 \mathrm{keV}$ for data taken prior to revolution 1000. The JEM-X data are limited to $\geqslant 5 \mathrm{keV}$ for similar reasons. Neither source is detected above $\sim 80 \mathrm{keV}$ in ISGRI or $\sim 30 \mathrm{keV}$ in JEM-X. We extracted spectra and light curves using version 10.0 of the standard Offline Scientific Analysis (OSA) software provided by the INTEGRAL Science Data Center (ISDC).

Coded-mask detectors such as those used by ISGRI and JEM-X work by deconvolving the "shadowgram" produced by opaque elements in the mask placed over the detector. While this allows imaging to be performed in the normally difficult hard $\mathrm{X}$-ray band, it also means the illuminated pixels in the detector receive light from every unocculted point in the FOV and as such every significant source in the FOV must be accounted for when determining the background for a given single source. We followed the recommendation of the ISDC and provided a catalog of all sources with detection significance greater than $6.0 \sigma$ in the single SCW's image. We provide the resulting list of sources in Table 1, along with their $20-40 \mathrm{keV}$ counting rate in the combined mosaic of all SCWs. The 20-40 keV ISGRI mosaic images from all SCWs are provided in Figure 1. 

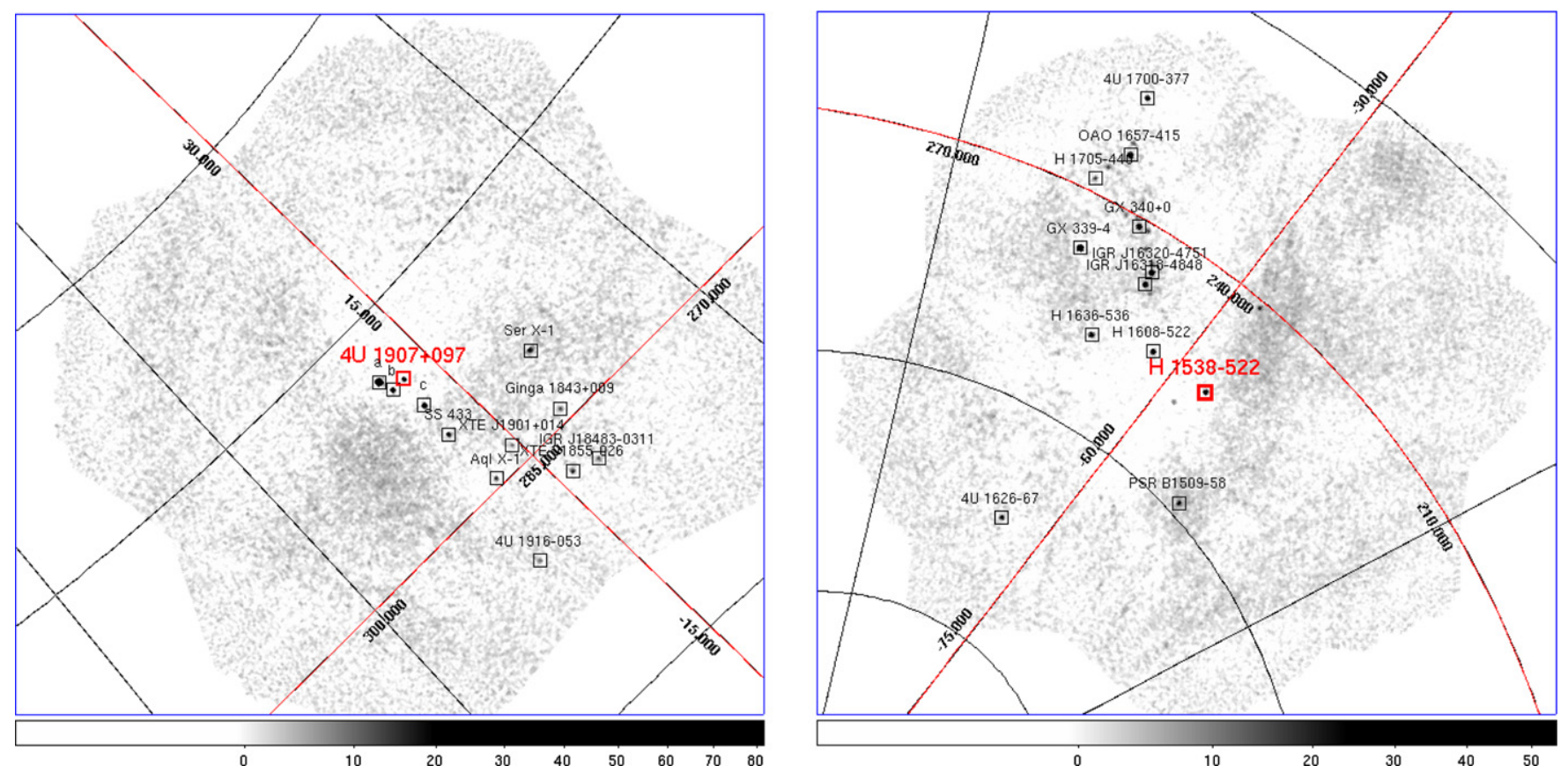

Figure 1. Combined 20-40 keV significance mosaics of the sky around $4 \mathrm{U} 1907+09$ (left) and 4U 1538-522 (right). Displayed coordinates are right ascension and declination in degrees. For clarity, the closest sources to 4U 1907+09 have been labeled with letters; they are (a) GRS 1915+105, (b) IGR J19140+0951, and (c) 4U 1909+07. While both images cover the full $27^{\circ} \times 27^{\circ}$ partially coded FOV of the ISGRI telescope, the SCWs that were selected to form these images all have the sources within the $9^{\circ} \times 9^{\circ}$ fully coded FOV. Both sources are clearly detected in the combined mosaics.

(A color version of this figure is available in the online journal.)

\section{1. $4 U 1538-522$}

Our ISGRI dataset comprises all publicly available data where 4U 1538-522 was within the 4.5 fully coded FOV, extending from 2003 January through 2010 April. The JEM-X data are selected in a similar fashion, taking all windows within the 2.4 radius fully coded FOV. There is a three year gap in the INTEGRAL coverage of 4U 1538-522, with no observations between late 2005 and late 2008. In the earlier data, the total ISGRI exposure is $715 \mathrm{ks}$, with 638 and $210 \mathrm{ks}$ for JEM-X 1 and 2 , respectively; the later dataset totals $417 \mathrm{ks}$ of ISGRI exposure, $411 \mathrm{ks}$ of JEM-X 1, and $172 \mathrm{ks}$ of JEM-X 2.

\section{2. $4 U 1907+09$}

We analyzed SCWs beginning in 2007 November (INTEGRAL revolution 608) for 4U 1907+09, overlapping slightly with the selection of Şahiner et al. (2012). Selecting SCWs according to the same criteria as used for 4U 1538-522 amounts to $892 \mathrm{ks}$ of ISGRI exposure, $908 \mathrm{ks}$ of JEM-X 1, and $507 \mathrm{ks}$ of JEM-X 2. The average flux from 4U 1907+09 is considerably lower than that of $4 \mathrm{U} 1538-522$ and is indeed at what appears to be close to a historic low for the source (comparable to that seen by Makishima et al. 1999 and Coburn 2001). As a result, it is difficult to provide strong constraints on its spectral parameters.

\section{TIMING ANALYSIS}

We extracted $10 \mathrm{~s}$ binned ISGRI light curves in the $20-40 \mathrm{keV}$ energy band with the OSA 10.0 analysis pipeline. The only departure from the standard procedure was the use of the alternate ii_light tool for light curve extraction, as the standard pipeline's light curve extraction routine is not recommended for binning times less than $60 \mathrm{~s}$ (Chernyakova et al. 2010). After
Table 2

Orbital Parameters

\begin{tabular}{lccc}
\hline \hline Parameter & Units & $4 \mathrm{U} 1538-522^{\mathrm{a}}$ & $4{\mathrm{U} 1907+09^{\mathrm{b}}}^{\circ}$ \\
\hline$a \sin (i)$ & lt-s & $53.1 \pm 1.5$ & $83 \pm 4$ \\
$e$ & $\ldots$ & $0.18 \pm 0.01$ & $0.28_{-0.14}^{+0.10}$ \\
$P_{\text {orb }}$ & day & $3.728382 \pm 0.000011$ & $8.3753_{-0.0002}^{+0.0003}$ \\
$T_{\pi / 2}$ & MJD & $52851.33 \pm 0.01$ & $50134.76_{-0.20}^{+0.16}$ \\
$\omega_{\mathrm{d}}$ & $\ldots$ & $40^{\circ} \pm 12^{\circ}$ & $330^{\circ} \pm 20^{\circ}$ \\
\hline
\end{tabular}

Notes.

a Mukherjee et al. (2006).

b in 't Zand et al. (1998).

extraction, the light curves were barycentered using the barycent tool also provided in the OSA package.

Due to the low signal to noise and few pulsations covered by the individual $\sim 2 \mathrm{ks}$ light curves, many light curves had to be appended to each other before any period determination could be made. The composite light curve was then corrected for the orbital motion of the source, using the best available orbital parameters. As the pulse profile of the source is non-sinusoidal and the composite light curve contains a moderate number of gaps, epoch folding (Leahy et al. 1983; Larsson 1996) was used for the period search, with $1 \sigma$ errors determined according to Larsson (1996).

\section{1. $4 U 1538-522$}

For 4U 1538-522, we used the elliptical orbital parameters provided by Mukherjee et al. (2006), although using Clark's (2000) circular solution did not result in any significant change in the pulse period. For convenience, these parameters are listed in Table 2. Light curves were grouped by requiring that no gaps larger than four days be present, in order to minimize the 


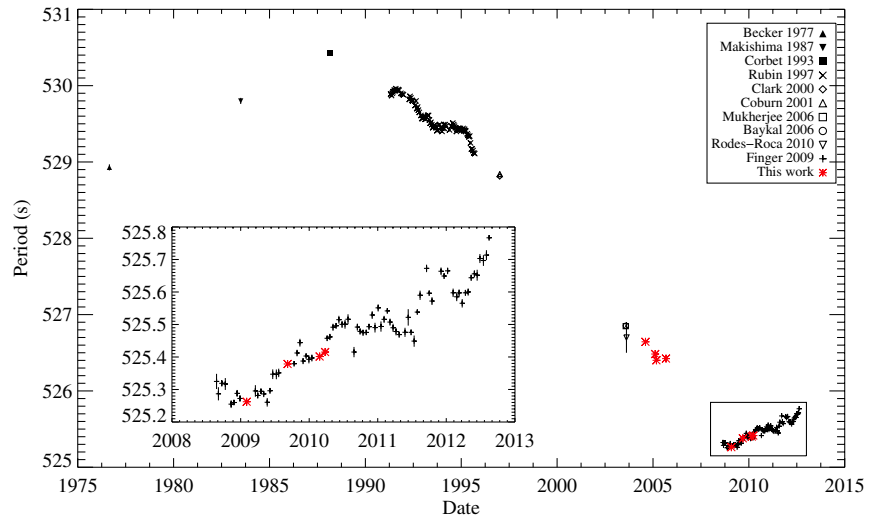

Figure 2. Pulse period evolution of 4 U 1538-522. Binary-corrected periods found in this work are represented by red asterisks, with previous measurements (Becker et al. 1977; Makishima et al. 1987; Corbet et al. 1993; Rubin et al. 1997; Clark 2000; Baykal et al. 2006) and recent Fermi GBM data (Finger et al. 2009) in black. Error bars are generally smaller than the data points. 4U 1538-522 evidently underwent a torque reversal sometime in late 2008 or early 2009 . The final four data points are in very good agreement with the Fermi GBM data; this agreement is detailed in the inset.

(A color version of this figure is available in the online journal.)

Table 3

Periods for 4U 1538-522 Determined via Epoch Folding

\begin{tabular}{lc}
\hline \hline MJD & Period (s) \\
\hline $53222.176-53230.047$ & $526.644 \pm 0.003$ \\
$53407.638-53415.310$ & $526.483 \pm 0.003$ \\
$53429.742-53439.492$ & $526.401 \pm 0.002$ \\
$53616.904-53620.899$ & $526.424 \pm 0.007$ \\
$54855.844-54870.322$ & $525.262 \pm 0.002$ \\
$55073.587-55090.517$ & $525.379 \pm 0.001$ \\
$55250.376-55258.045$ & $525.401 \pm 0.004$ \\
$55264.806-55288.806$ & $525.415 \pm 0.001$ \\
\hline
\end{tabular}

effect of large gaps in the data when performing epoch folding. Eight sets of SCWs using this grouping have sufficient time and statistics to return clear single peaks in the epoch folding results; we summarize these results in Table 3 and the source's pulse period history is updated in Figure 2. Pulse periods from the earlier data, prior to 2008, follow roughly the same spin-up trend as originally seen by Rubin et al. (1997), with the period dropping at a rate of $\sim 0.2 \mathrm{~s} \mathrm{yr}^{-1}$. However, our measured pulse periods post-2008 reveal a new spin-down trend of $\sim 0.13 \mathrm{~s} \mathrm{yr}^{-1}$. This conclusion is supported by the results of the Fermi GBM Pulsar Project (Finger et al. 2009), ${ }^{7}$ which we also include in Figure 2. The pulse shape (see Figure 3) in the $20-40 \mathrm{keV}$ band is dominantly single-peaked; the disappearance of the secondary peak in the $\gtrsim 20 \mathrm{keV}$ pulse profile has been seen before by Clark et al. (1990) and Robba et al. (2001), who suggested it may be due to the presence of the $22 \mathrm{keV}$ CRSF. The pulse profiles from all observations do not vary significantly from group to group, with the average difference between profiles generally $20 \%$ to $50 \%$ smaller than the average noise in the profiles. This persists across the torque reversal.

\section{2. $4 U 1907+09$}

We began our 4U 1907+09 timing analysis with data from INTEGRAL revolution 608, roughly where the INTEGRAL analysis of Şahiner et al. (2012) ended. The data were binary

\footnotetext{
7 Results for $4 \mathrm{U} 1538-522$ can be found at

http://gammaray.nsstc.nasa.gov/gbm/science/pulsars.
}

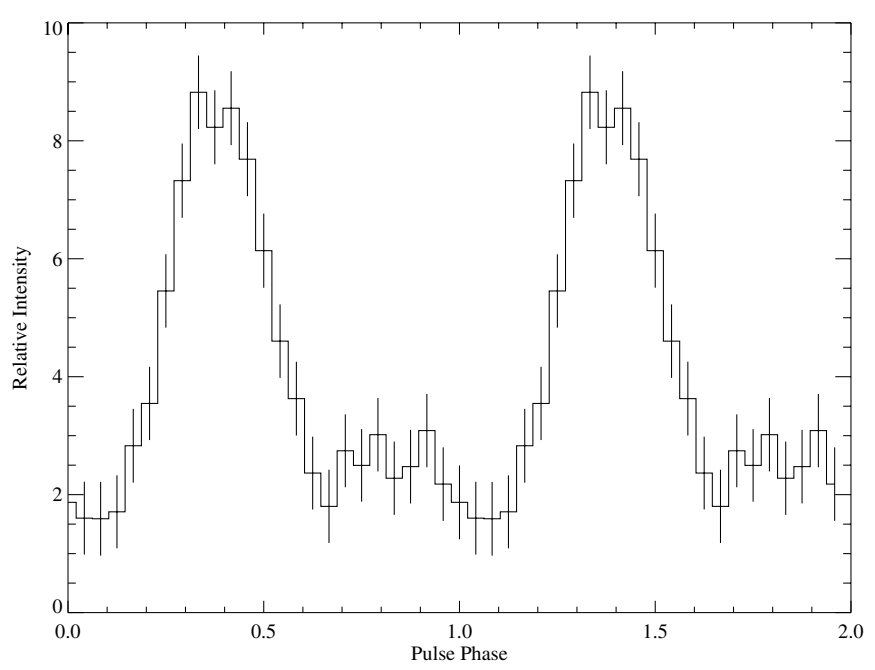

Figure 3. Typical $20-40 \mathrm{keV}$ pulse profile for $4 \mathrm{U}$ 1538-522. The average pulse shape is strongly single-peaked in this energy band. This profile is for the first epoch listed in Table 3; the variations between pulse profiles for the different epochs listed are not statistically significant.

corrected according to the parameters determined by in 't Zand et al. (1998), listed in Table 2. During the time encompassed by our analysis, the source was considerably fainter when compared with previous studies using INTEGRAL (Fritz et al. 2006; Şahiner et al. 2012) and was significantly ( 40\%) dimmer than $4 \mathrm{U} 1538-522$ (compare the $5-100 \mathrm{keV}$ fluxes for $4 \mathrm{U}$ $1538-522$ in Tables 4 and 5 with the equivalent flux for $4 \mathrm{U}$ 1907+09 in Table 6). When epoch folding, no selection of SCWs from the new INTEGRAL data produce $\chi^{2}$ distributions that are comparable with our results for $4 \mathrm{U} 1538-522$ and all errorestimation procedures return errors of $\sim 1 \mathrm{~s}$, compared with $\sim 0.001 \mathrm{~s}$ for $4 \mathrm{U} 1538-522$. As a consistency check, SCWs from INTEGRAL revolutions 608 through 623, already reported by Şahiner et al. (2012), were analyzed in the same manner; for this data subset alone, the epoch folding returns a statistically significant result that is consistent with Şahiner et al.'s. We thus do not report any INTEGRAL-derived pulse periods for $4 \mathrm{U}$ $1907+09$ due to the faintness of the source.

\section{SPECTRAL ANALYSIS}

We extracted ISGRI and JEM-X spectra for 4U 1538-522 and $4 \mathrm{U} 1907+09$ on a SCW-by-SCW basis using the OSA 10.0 software, combining the individual SCW spectra with the spe_pick tool provided in the OSA package. The extracted spectra were then modeled in XSPEC version 12.7.1 (Arnaud 1996). All error bars are for the $90 \%$ single-parameter confidence interval unless otherwise indicated and 5-100 keV model fluxes with $90 \%$ error bars were computed using XSPEC. The spectra for both sources descend into noise above $\sim 50-80 \mathrm{keV}$, depending on the particular subset of data being analyzed. Spectra were regrouped to provide roughly similar error bars for data above the noise threshold.

We present spectral fits for two continuum models. The PLCUT model (in XSPEC, POWERLAW*HIGHECUT) is a piecewise function, with a power law multiplied by a high energy exponential cutoff above some cutoff energy $E_{\text {cut }}$ :

$$
\operatorname{PLCUT}(E)= \begin{cases}A \times E^{-\Gamma} & E<E_{\text {cut }} \\ A \times E^{-\Gamma} \exp \left(\frac{E_{\text {cut }}-E}{E_{\text {fold }}}\right) & E \geqslant E_{\text {cut }},\end{cases}
$$


Table 4

Spectral Parameters for 4U 1538-522 for 2003-2008 and 2008-2010

\begin{tabular}{|c|c|c|c|c|c|}
\hline \multirow[b]{2}{*}{ Continuum } & & \multicolumn{2}{|c|}{ Early (2003-2008) } & \multicolumn{2}{|c|}{ Late (2008-2010) } \\
\hline & & POWERLAW & CUTOFFPL+BUMP & POWERLAW & CUTOFFPL+BUMP \\
\hline $\bar{\Gamma}$ & & $1.17_{-0.07}^{+0.06}$ & $0.7_{-0.2}^{+0.2}$ & $1.12_{-0.14}^{+0.08}$ & $0.4_{-0.5}^{+0.2}$ \\
\hline Normalization & a & $3.3_{-0.5}^{+0.6}$ & $2.2_{-0.7}^{+0.8}$ & $2.9_{-0.7}^{+0.6}$ & $1.6_{-0.7}^{+0.5}$ \\
\hline 5-100 keV flux & $\mathrm{b}$ & $6.62_{-3.36}^{+0.08}$ & $6.48_{-0.40}^{+0.14}$ & $6.3_{-0.6}^{+0.2}$ & $6.3_{-0.5}^{+0.2}$ \\
\hline$E_{\text {cut }}$ & $\mathrm{keV}$ & $14.2_{-1.0}^{+1.3}$ & $\ldots$ & $14_{-2}^{+3}$ & $\ldots$ \\
\hline$E_{\text {fold }}$ & $\mathrm{keV}$ & $14_{-2}^{+4}$ & $13_{-2}^{+3}$ & $11.7_{-1.5}^{+1.3}$ & $9.4_{-1.6}^{+1.0}$ \\
\hline$E_{\text {bump }}$ & $\mathrm{keV}$ & $\ldots$ & $13.0_{-0.5}^{+0.8}$ & $\ldots$ & $13.3_{-0.8}^{+1.0}$ \\
\hline$\sigma_{\text {bump }}$ & $\mathrm{keV}$ & $\cdots$ & $1.7_{-0.3}^{+0.8}$ & $\ldots$ & 1.70 (frozen) \\
\hline Intensity $_{\text {bump }}$ & $\mathrm{c}$ & $\ldots$ & $1.4_{-0.6}^{+1.3}$ & $\ldots$ & $1.2_{-0.6}^{+2.4}$ \\
\hline$E_{\mathrm{c} 1}$ & $\mathrm{keV}$ & $21.5_{-0.6}^{+0.5}$ & $22.0_{-1.8}^{+0.6}$ & $21.1_{-0.8}^{+0.8}$ & $21.9_{-3.9}^{+0.8}$ \\
\hline$\sigma_{\mathrm{c} 1}$ & $\mathrm{keV}$ & $3.2_{-0.6}^{+0.7}$ & $2.9_{-1.1}^{+1.9}$ & $3.8_{-1.2}^{+1.4}$ & $3.4_{-1.2}^{+5.0}$ \\
\hline$\tau_{\mathrm{c} 1}$ & & $0.48_{-0.07}^{+0.11}$ & $0.32_{-0.08}^{+0.09}$ & $0.46_{-0.13}^{+0.21}$ & $0.38_{-0.10}^{+0.11}$ \\
\hline$E_{\mathrm{c} 2}$ & $\mathrm{keV}$ & $50_{-4}^{+5}$ & $50_{-3}^{+4}$ & $\cdots$ & $\cdots$ \\
\hline$\sigma_{\mathrm{c} 2}$ & $\mathrm{keV}$ & $8_{-3}^{+6}$ & $8_{-2}^{+3}$ & $\cdots$ & $\cdots$ \\
\hline$\tau_{\mathrm{c} 2}$ & & $0.8_{-0.3}^{+0.6}$ & $1.0_{-0.3}^{+0.4}$ & $\ldots$ & $\ldots$ \\
\hline JEM-X 1 normalization & & $0.91_{-0.06}^{+0.06}$ & $0.96_{-0.06}^{+0.06}$ & $0.96_{-0.10}^{+0.11}$ & $0.92_{-0.09}^{+0.09}$ \\
\hline JEM-X 2 normalization & & $1.02_{-0.07}^{+0.07}$ & $1.07_{-0.07}^{+0.07}$ & $1.07_{-0.11}^{+0.12}$ & $1.03_{-0.10}^{+0.10}$ \\
\hline$\chi_{\text {red }}^{2}($ dof $)$ & & $1.175(44)$ & $1.095(43)$ & $0.7277(46)$ & $0.6633(45)$ \\
\hline
\end{tabular}

Notes.

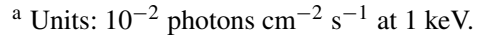

b Units: $10^{-10} \mathrm{erg} \mathrm{cm}^{-2} \mathrm{~s}^{-1}$.

${ }^{\mathrm{c}}$ Units: $10^{-3}$ photons $\mathrm{cm}^{-2} \mathrm{~s}^{-1}$.

where $A$ is the model flux in erg $\mathrm{cm}^{-2} \mathrm{~s}^{-1}$ at $1 \mathrm{keV}$ and $E_{\text {fold }}$ is the $e$-folding energy that turns on at energies higher than the cutoff energy $E_{\text {cut }}$. The CUTOFFPL continuum is PLCUT with the $E_{\text {cut }}$ parameter frozen at zero. Since this means the CUTOFFPL spectrum is being exponentially attenuated at all energies, it generally has a significantly lower photon index $\Gamma$, but its continuous nature means it lacks the sharp corner that in PLCUT can produce spurious residuals around the cutoff energy. However, CUTOFFPL on its own results in high $\chi^{2}$ values for spectral fits in some cases, requiring an additional broad Gaussian component to be added to the spectral model. A feature like this at $\sim 10 \mathrm{keV}$ has been seen before in both $4 \mathrm{U} 1538-522$ (Coburn 2001) and 4U 1907+09 (Mihara 1995; Coburn 2001; Rivers et al. 2010) as an emission feature, as well as many other HMXBs both with and without cyclotron features (although it occasionally can be modeled as an absorption feature; see, e.g., Müller et al. 2012) and is likely due to the simplistic nature of our continuum models in light of the complex physics of the actual accretion column.

There are two other commonly used continuum models, the Fermi-Dirac cutoff model FDCUT and the two power law NPEX, but neither model was capable of producing a stable fit in either source, generally returning many unconstrained parameters. We did not incorporate any photoelectric absorption, as it is not strongly pronounced at energies above a few $\mathrm{keV}$, we did not include the Fe $\mathrm{K}$ line at $6.4 \mathrm{keV}$ (Rodes-Roca et al. 2010) either, as the energy binning needed to obtain usable statistics is too coarse to observe the line.

Both sources exhibit absorption-like features in the $\sim 20$ and $\sim 40-50 \mathrm{keV}$ range. These are typically identified as CRSFs, and a brief description of the mechanism for their production is presented in Section 5. They typically appear as broad
( $\sigma \gtrsim 3 \mathrm{keV}$ ), relatively shallow absorption-like features, usually modeled by multiplicative Gaussians or pseudo-Lorentzians with negative intensity. We use a local XSPEC model, GAUABS, a Gaussian optical depth profile defined by

$$
\begin{gathered}
\operatorname{GAuABS}(\tau)=F_{0} e^{-\tau(E)} \\
\tau(E)=\tau_{0} \exp \left(-\frac{\left(E-E_{0}\right)^{2}}{2 \sigma^{2}}\right) .
\end{gathered}
$$

The broad width and shallowness of these lines makes their detection and constraint difficult in the exponentially dropping spectra of HMXBs, especially when it comes to the higher harmonics that lie far above the cutoff energy of the spectrum. In particular, the width of the harmonic was unconstrained in some cases and we opted to fix it at its fitted value when determining errors. In very low-flux datasets, the harmonic CRSF is often not detected.

\section{1. $4 U 1538-522$ \\ 4.1.1. Date-selected Spectra}

The three year gap in INTEGRAL coverage of 4U 1538-522, along with the presence of the torque reversal of late 2008/early 2009 , presents an obvious point at which to split the dataset, so we analyze the 2003-2005 and 2008-2010 spectra separately.

Each dataset was fit with the two aforementioned continuum models. Each continuum was modified by one or two Gaussianprofile absorption features and a constant multiplier, which was frozen at unity for the ISGRI spectra and allowed to vary for the JEM-X spectra in order to account for calibration differences between the instruments. The two continuum models provide 
Table 5

Spectral Parameters for 4U 1538-522 for Three Luminosity Bins

\begin{tabular}{|c|c|c|c|c|c|c|c|c|c|c|c|c|c|}
\hline \multirow[b]{3}{*}{ Continuum } & & \multicolumn{4}{|c|}{ High } & \multicolumn{4}{|c|}{ Mid } & \multicolumn{4}{|c|}{ Low } \\
\hline & & \multicolumn{2}{|c|}{ Early } & \multicolumn{2}{|c|}{ Late } & \multicolumn{2}{|c|}{ Early } & \multicolumn{2}{|c|}{ Late } & \multicolumn{2}{|c|}{ Early } & \multicolumn{2}{|c|}{ Late } \\
\hline & & $P^{\mathrm{a}}$ & $C^{\mathrm{b}}$ & $P$ & C & $P$ & $\mathrm{CB}^{\mathrm{c}}$ & $P$ & $C$ & $P$ & C & $P$ & C \\
\hline $\bar{\Gamma}$ & & $1.07_{-0.16}^{+0.10}$ & $0.3_{-0.2}^{+0.2}$ & $1.04_{-0.27}^{+0.12}$ & $0.4_{-0.3}^{+0.2}$ & $1.17_{-0.07}^{+0.07}$ & $0.9_{-0.2}^{+0.2}$ & $1.06_{-0.17}^{+0.09}$ & $0.6_{-0.2}^{+0.2}$ & $1.20_{-0.14}^{+0.12}$ & $0.2_{-0.2}^{+0.2}$ & $1.18_{-0.14}^{+0.12}$ & $0.1_{-0.2}^{+0.2}$ \\
\hline Normalization & d & $4.8_{-1.3}^{+1.2}$ & $2.1_{-0.5}^{+0.6}$ & $4.6_{-1.6}^{+1.3}$ & $2.4_{-0.7}^{+0.9}$ & $3.7_{-0.6}^{+0.7}$ & $3.6_{-1.1}^{+1.5}$ & $3.0_{-0.4}^{+0.7}$ & $1.8_{-0.6}^{+0.7}$ & $1.9_{-0.5}^{+0.6}$ & $0.6_{-0.2}^{+0.3}$ & $2.1_{-0.6}^{+0.8}$ & $0.7_{-0.2}^{+0.3}$ \\
\hline 5-100 keV flux & $\mathrm{e}$ & $11.4_{-1.4}^{+0.2}$ & $11.4_{-2.8}^{+0.2}$ & $9.9_{-2.4}^{+0.3}$ & $10.1_{-2.5}^{+0.2}$ & $7.1_{-0.4}^{+0.2}$ & $7.1_{-1.0}^{+0.2}$ & $8_{-2}^{+6}$ & $8.3_{-1.4}^{+0.3}$ & $3.2_{-0.4}^{+0.2}$ & $3.22_{-0.27}^{+0.07}$ & $3.9_{-0.4}^{+0.3}$ & $4.0_{-0.7}^{+0.2}$ \\
\hline$E_{\text {cut }}$ & $\mathrm{keV}$ & $14_{-2}^{+2}$ & $\ldots$ & $13_{-2}^{+4}$ & $\ldots$ & $13.8_{-1.2}^{+1.8}$ & .. & $13_{-2}^{+2}$ & .. & $22_{-4}^{+5}$ & $\ldots$ & $14_{-2}^{+7}$ & .. \\
\hline$E_{\text {fold }}$ & $\mathrm{keV}$ & $13_{-2}^{+2}$ & $11_{-2}^{+2}$ & $18_{-4}^{+4}$ & $15_{-3}^{+3}$ & $14_{-2}^{+2}$ & $14_{-3}^{+4}$ & $>13.0$ & $14_{-4}^{+4}$ & $8_{-2}^{+2}$ & $8.2_{-0.8}^{+1.0}$ & $12_{-4}^{+2}$ & $8.1_{-1.1}^{+1.3}$ \\
\hline$E_{\text {bump }}$ & $\mathrm{keV}$ & $\ldots$ & $\ldots$ & $\ldots$ & $\ldots$ & $\ldots$ & $12.7_{-0.6}^{+1.0}$ & $\ldots$ & $\ldots$ & $\ldots$ & $\ldots$ & $\ldots$ & $\ldots$ \\
\hline$\sigma_{\text {bump }}$ & $\mathrm{keV}$ & $\ldots$ & $\ldots$ & $\ldots$ & $\ldots$ & $\ldots$ & 2.03 (frozen) & $\ldots$ & $\ldots$ & $\ldots$ & $\ldots$ & $\ldots$ & $\ldots$ \\
\hline Intensity bump & f & $\ldots$ & $\ldots$ & $\ldots$ & $\ldots$ & $\ldots$ & $2.6_{-0.9}^{+1.0}$ & $\ldots$ & .. & $\ldots$ & $\ldots$ & $\ldots$ & $\ldots$ \\
\hline$E_{\mathrm{c} 1}$ & $\mathrm{keV}$ & $21.2_{-0.7}^{+0.6}$ & $22.0_{-0.4}^{+0.5}$ & $21.6_{-1.1}^{+1.0}$ & $21.9_{-0.9}^{+0.9}$ & $21.5_{-0.7}^{+0.7}$ & $22.1_{-0.9}^{+0.7}$ & $23.1_{-1.3}^{+1.1}$ & $24.1_{-1.3}^{+1.5}$ & $21.8_{-1.4}^{+2.3}$ & $21.3_{-0.7}^{+0.8}$ & $21.5_{-1.1}^{+1.2}$ & $21.9_{-1.0}^{+1.2}$ \\
\hline$\sigma_{\mathrm{c} 1}$ & $\mathrm{keV}$ & $3.7_{-1.1}^{+1.1}$ & $3.4_{-0.7}^{+0.7}$ & $4.0_{-1.2}^{+1.5}$ & $3.8_{-1.0}^{+1.2}$ & $3.3_{-0.9}^{+1.0}$ & $2.8_{-1.4}^{+2.1}$ & $5.6_{-1.6}^{+1.0}$ & $5_{-2}^{+2}$ & $3.4_{-0.9}^{+0.8}$ & $2.2_{-1.5}^{+1.1}$ & $2_{-2}^{+3}$ & $1.6_{-1.4}^{+1.6}$ \\
\hline$\tau_{\mathrm{c} 1}$ & & $0.56_{-0.13}^{+0.22}$ & $0.60_{-0.13}^{+0.12}$ & $0.6_{-0.2}^{+0.2}$ & $0.6_{-0.2}^{+0.2}$ & $0.45_{-0.11}^{+0.13}$ & $0.40_{-0.11}^{+0.13}$ & $0.6_{-0.3}^{+0.2}$ & $0.65_{-0.10}^{+0.23}$ & $1.0_{-0.3}^{+0.3}$ & $0.6_{-0.2}^{+1.2}$ & $0.8_{-0.4}^{+7.9}$ & $>0.3$ \\
\hline$E_{\mathrm{c} 2}$ & $\mathrm{keV}$ & $49_{-3}^{+4}$ & $49_{-3}^{+4}$ & $50_{-4}^{+8}$ & $49_{-4}^{+7}$ & $50_{-3}^{+4}$ & $53_{-3}^{+4}$ & $57_{-13}^{+6}$ & $53_{-4}^{+4}$ & $\ldots$ & $\ldots$ & $\ldots$ & $\ldots$ \\
\hline$\sigma_{\mathrm{c} 2}$ & $\mathrm{keV}$ & $8_{-3}^{+4}$ & $10_{-4}^{+3}$ & $15_{-4}^{+14}$ & $16_{-4}^{+11}$ & 8 (frozen) & 10 (frozen) & $14_{-11}^{+5}$ & 10 (frozen) & $\ldots$ & $\ldots$ & $\ldots$ & $\ldots$ \\
\hline JEM-X 1 normalization & & $0.99_{-0.07}^{+0.07}$ & $1.01_{-0.09}^{+0.10}$ & $0.87_{-0.12}^{+0.13}$ & $0.89_{-0.11}^{+0.13}$ & $0.99_{-0.09}^{+0.10}$ & $0.93_{-0.08}^{+0.08}$ & $1.12_{-0.13}^{+0.08}$ & $1.14_{-0.12}^{+0.14}$ & $0.97_{-0.10}^{+0.11}$ & $0.94_{-0.10}^{+0.11}$ & $1.0_{-0.2}^{+0.2}$ & $1.1_{-0.2}^{+0.2}$ \\
\hline JEM-X 2 normalization & & $0.72_{-0.05}^{+0.05}$ & $0.73_{-0.07}^{+0.07}$ & $0.89_{-0.13}^{+0.13}$ & $0.91_{-0.12}^{+0.13}$ & $0.93_{-0.09}^{+0.10}$ & $0.87_{-0.08}^{+0.08}$ & $1.23_{-0.14}^{+0.14}$ & $\begin{array}{l}1.25_{-0.14}^{+0.15} \\
\end{array}$ & $1.3_{-0.2}^{+0.2}$ & $\begin{array}{l}1.31_{-0.15}^{+0.16} \\
\end{array}$ & $1.1_{-0.2}^{+0.2}$ & $1.1_{-0.2}^{+0.2}$ \\
\hline$\overline{\chi_{\text {red }}^{2}(\text { dof })}$ & & $1.038(54)$ & $1.134(55)$ & $0.7066(50)$ & $0.7644(51)$ & $1.060(46)$ & $1.090(45)$ & $0.8842(50)$ & $0.9487(52)$ & $1.224(41)$ & $1.356(42)$ & $0.4921(41)$ & $0.5870(42)$ \\
\hline
\end{tabular}

Notes.

a PLCUT continuum.

${ }^{\mathrm{b}}$ CUTOFFPL continuum.

${ }^{c}$ CUTOFFPL+BUMP continuum.

${ }^{\mathrm{d}}$ Units: $10^{-2}$ photons $\mathrm{cm}^{-2} \mathrm{~s}^{-1}$ at $1 \mathrm{keV}$.

${ }^{\mathrm{e}}$ Units: $10^{-10} \mathrm{erg} \mathrm{cm}^{-2} \mathrm{~s}^{-1}$

${ }^{\mathrm{f}}$ Units: $10^{-3}$ photons $\mathrm{cm}^{-2} \mathrm{~s}^{-1}$. 

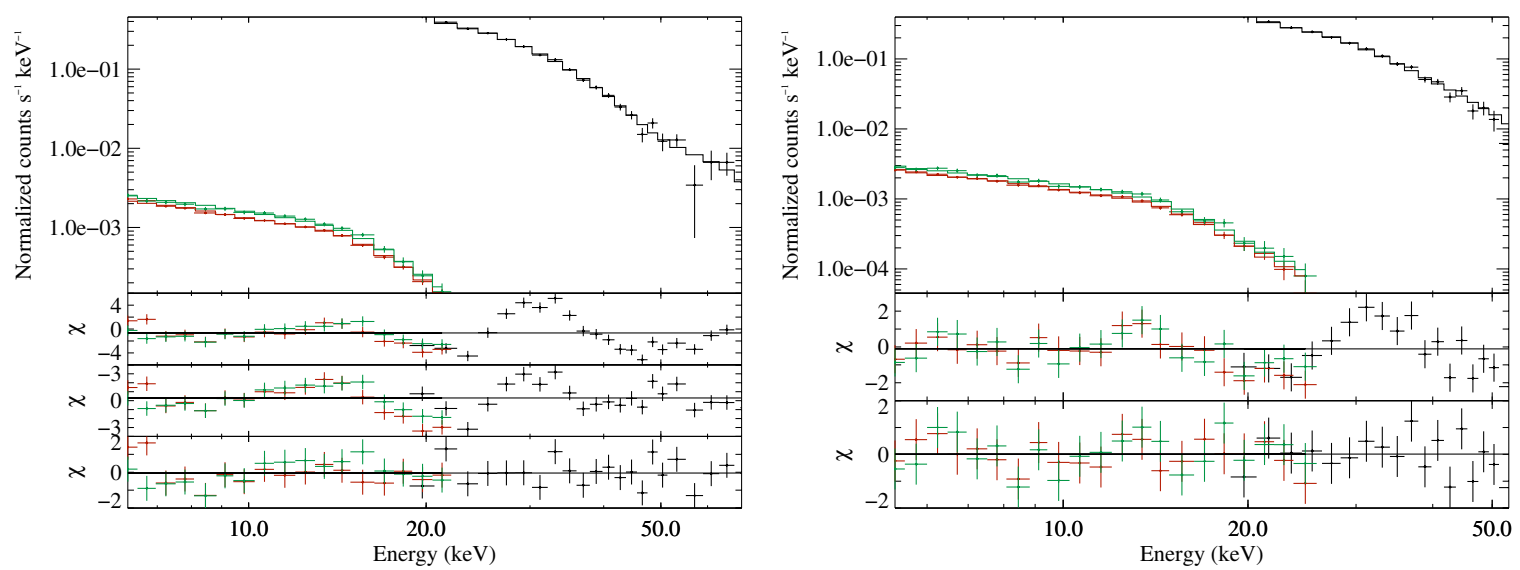

Figure 4. Date-selected counts spectra and models for 4 U 1538-522 using the PLCUT continuum. The black points indicate the ISGRI spectrum; the red and green indicate JEM-X 1 and 2, respectively. The left panel displays the earlier dataset, comprising data from 2003 to 2008, with residuals for the PLCUT continuum with, from top to bottom, no CRSFs, one CRSF at $\sim 50 \mathrm{keV}$, and CRSFs at $21.5 \mathrm{keV}$ and $50 \mathrm{keV}$. The right panel contains the later dataset, spanning the years $2008-2010$, and the residuals are for the continuum with no CRSFs (top) and one CRSF at $21.1 \mathrm{keV}$ (bottom).

(A color version of this figure is available in the online journal.)

Table 6

Spectral Parameters for 4U $1907+09$

\begin{tabular}{lccc}
\hline \hline Continuum & & POWERLAW & CUTOFFPL+BUMP \\
\hline$\Gamma$ & & $-0.9_{-0.8}^{+0.8}$ & $-1.3_{-0.7}^{+1.0}$ \\
Normalization & $\mathrm{a}$ & $0.03_{-0.02}^{+0.09}$ & $0.04_{-0.03}^{+0.1}$ \\
$5-100 \mathrm{keV}$ flux & $\mathrm{b}$ & $2.92_{-0.31}^{+0.04}$ & $2.92_{-0.20}^{+0.05}$ \\
$E_{\text {cut }}$ & $\mathrm{keV}$ & $6.5_{-2.4}^{+1.6}$ & $\ldots$ \\
$E_{\text {fold }}$ & $\mathrm{keV}$ & $6.2_{-1.3}^{+3.8}$ & $5.6_{-1.2}^{+17.2}$ \\
\hline$E_{\mathrm{c} 1}$ & $\mathrm{keV}$ & $18.5_{-0.7}^{+0.7}$ & $18.4_{-0.7}^{+0.7}$ \\
$\sigma_{\mathrm{c} 1}$ & $\mathrm{keV}$ & $3.2_{-0.8}^{+1.1}$ & $3.5_{-0.8}^{+1.1}$ \\
$\tau_{\mathrm{c} 1}$ & & $0.8_{-0.2}^{+0.2}$ & $0.9_{-0.2}^{+0.3}$ \\
\hline$E_{\mathrm{c} 2}$ & $\mathrm{keV}$ & $38_{-5}^{+8}$ & $38_{-5}^{+14}$ \\
$\sigma_{\mathrm{c} 2}$ & $\mathrm{keV}$ & $8_{-6}^{+14}$ & $9_{-5}^{+18}$ \\
$\tau_{\mathrm{c} 2}$ & & $1.1_{-0.8}^{+5.4}$ & $1.2_{-0.9}^{+5.1}$ \\
\hline JEM-X 1 normalization & & $0.95_{-0.12}^{+0.13}$ & $0.95_{-0.12}^{+0.13}$ \\
JEM-X 2 normalization & & $1.19_{-0.15}^{+0.16}$ & $1.19_{-0.15}^{+0.16}$ \\
\hline$\chi_{\text {red }}^{2}($ dof) & & $0.7774(35)$ & $0.8260(36)$ \\
\hline
\end{tabular}

Notes.

a Units: $10^{-2}$ photons $\mathrm{cm}^{-2} \mathrm{~s}^{-1}$ at $1 \mathrm{keV}$.

${ }^{b}$ Units: $10^{-10} \mathrm{erg} \mathrm{cm}^{-2} \mathrm{~s}^{-1}$.

similar $\chi^{2}$ statistics, with CUTOFFPL+BUMP being slightly superior in most cases. However, when calculating errors, it was necessary to fix the width of the $10 \mathrm{keV}$ feature in the later dataset at the value found in the earlier dataset, as the decreasing sensitivity of the JEM-X telescopes at higher energies, combined with the presence of the CRSF at $\sim 22 \mathrm{keV}$, resulted in unconstrained fit parameters. We will refer primarily to the PLCUT continuum results in our discussion due to this limitation. Neither continuum model shows any significant variation across the torque reversal. We list the parameters for the spectral fits for both continua in Table 4 and the spectra for the PLCUT continuum are plotted in Figure 4.

We clearly detect the fundamental CRSF at $21.5_{-0.6}^{+0.5} \mathrm{keV}$ in the early dataset and $21.1 \pm 0.8 \mathrm{keV}$ in the late dataset. Adding the feature in the early spectra decreases the $\chi^{2}$ per degree of freedom (dof) statistic from $327 / 50$ to $70 / 47$, while in the late dataset, $\chi^{2} /$ dof drops from $84 / 49$ to $33 / 46$. There are no statistically significant differences in the fundamental CRSF across the torque reversal. The harmonic CRSF is much less significantly detected in the early dataset: its addition at $50_{-4}^{+5} \mathrm{keV}$ lowers the $\chi^{2} /$ dof to $52 / 44$. In the later data, the feature is not detected; adding a $\sim 50 \mathrm{keV}$ GAUABS component reduces the $\chi^{2}$ by a negligible amount and generally has unconstrained width and depth, so we leave the feature out of our model for the late data. This non-detection is likely a product of the fact that we are adding together all spectra from after the torque reversal and the lower luminosity observations are driving down the signal-to-noise ratio. This can be more clearly seen in the following section.

\subsubsection{Luminosity-selected Spectra}

To more closely examine the differences between the earlier and later spectra for $4 \mathrm{U} 1538-522$, we further split the dateselected SCWs into high-, middle-, and low-luminosity bins by taking a $1 \sigma$ region around the average brightness $\mathrm{SCW}$ to be the middle-luminosity bin. The 5-100 keV flux of the source varies by a factor of $\sim 3$ from the lowest to highest luminosity bin. The best-fit parameters for these datasets for two continuum models are presented in Table 5. Both continuum models again provide roughly similar $\chi^{2}$ statistics for each dataset. The earlier mid-luminosity bin was the only dataset in the luminosityselected data that showed a clear need for a $10 \mathrm{keV}$ feature; however, it was necessary to freeze the width of the feature at its fitted value to obtain a stable fit, due to the interaction between the $22 \mathrm{keV}$ CRSF and the "bump." We additionally froze the width of the harmonic CRSF in all but one model for the mid-luminosity datasets, as a dearth of counts at high energy often led this parameter to be unconstrained. In these cases, we froze the value of the width to the value found in the same model for the corresponding high-luminosity dataset, the exception being the later mid-luminosity spectrum, where the $16 \mathrm{keV}$ width of the later high-luminosity data did not produce a good fit. In that case, the width was frozen to the value found in the earlier high-luminosity data. The harmonic CRSF was not detected in the low-luminosity dataset. 
In the PLCUT continuum, $E_{\text {fold }}$ is the only parameter showing a significant correlation with luminosity, with a slope of $1.2 \pm$ $0.8 \mathrm{keV} /\left(10^{36} \mathrm{erg} \mathrm{s}^{-1}\right)(90 \%$ confidence $)$. The correlation is significant at the $p=0.02$ level. The other spectral parameters generally do not show clearly significant trends; $\Gamma$ and $E_{\text {cut }}$ have slight correlations, but only at the $1 \sigma$ level. The missing harmonic CRSF is consistent with a lack of statistics at energies above $\sim 40 \mathrm{keV}$ in the low-luminosity datasets; attempting to fit a line with energy and width frozen at the value from the midluminosity bin results in an unbounded depth, and so we do not include the harmonic CRSF in the model for the low-luminosity data.

\section{2. $4 U 1907+09$}

The SCWs for 4U 1907+09 are overall more evenly spread throughout INTEGRAL's mission, with no significant changes in pulse period derivative, so there is no logical point in time to define a date-based separation as we have done for $4 \mathrm{U}$ 1538-522. The best-fit spectral parameters for all analyzed 4U 1907+09 SCWs are presented in Table 6, with the folded spectrum and model residuals for the PLCUT continuum plotted in Figure 5. Previous analyses (Fritz et al. 2006; Rivers et al. 2010; Şahiner et al. 2012) have been performed using the PLCUT, FDCUT, and NPEX continua, but as is stated in Section 4, we could not obtain useful fits for the latter two. There was no evidence in the residuals for a bump feature at $\sim 13 \mathrm{keV}$ as has been seen in spectra from, e.g., Suzaku (Rivers et al. 2010); an attempt at adding in the feature produced minimal $(\ll 1)$ improvements in $\chi^{2}$ along with very poorly constrained energy, width, and height. Without the feature, the CUTOFFPL continuum produces equally acceptable results compared with the PLCUT continuum.

The addition of the fundamental CRSF at $\sim 18 \mathrm{keV}$ improves the fit significantly, lowering the $\chi^{2}$ by $\sim 100$. This feature can be seen primarily in the JEM-X residuals, while ISGRI, with its $20 \mathrm{keV}$ lower bound, can only resolve its upper edge. The harmonic is much less significantly detected at $\sim 38 \mathrm{keV}$, producing a drop of $\sim 10$ in $\chi^{2}$ in both models. Adding the harmonic without the fundamental produces a drop of $\sim 20$. While the spectrum is already well fit with only one CRSF, we include both in our final models on account of their wellestablished existence in the literature (Cusumano et al. 1998; Makishima et al. 1999; Fritz et al. 2006; Rivers et al. 2010) and the fact that we are still capable of constraining the harmonic CRSF. Our measurement of the fundamental CRSF is compatible with the measurements by Makishima et al. (1999) and Coburn (2001), which were made using observations of the source at a similar flux, although, as will be discussed in Section 5, Makishima et al.'s measurement was made using a different model for the line, which could make that result inconsistent with ours.

An attempt was made to split 4U 1907+09's spectra into luminosity bins as we did for $4 \mathrm{U} 1538-522$, but the poorer statistics for 4U 1907+09 made this unfeasible, with satisfactory fits only obtained for the brightest luminosity bin. This difficulty is illustrated by the considerably lower 5-100 keV flux of $4 \mathrm{U}$ $1907+09$ compared with that of 4 U 1538-522 - the overall flux from the full $4 \mathrm{U} 1907+09$ dataset was $\sim 40 \%$ lower than the flux for 4U 1538-522 and was in fact considerably lower than the flux from our lowest-luminosity dataset for 4U 1538-522 (compare Table 6 with Tables 4 and 5).

\section{DISCUSSION}

\subsection{Torque Reversal}

Our pulse period measurements for 4U 1538-522 show evidence for a torque reversal in late 2008 or early 2009, although no significant spectral changes are detected that could shed light on a mechanism for this change. Pulse period evolution in accreting neutron stars like $4 \mathrm{U} 1538-522$ is primarily driven by the interaction between the accreting material and the magnetic field of the star. As the field lines are locked into the neutron star, they rotate with the star and the infalling material can exert a torque on the star. Changes in the accretion rate can thus produce changes in the pulse period, but the relationship between accretion rate and torque can be very complex due to the interaction between the accreting material, which may or may not form an accretion disk, and the magnetic field of the neutron star. $4 \mathrm{U} 1538-522$ is primarily a wind-accretor, as the main-sequence companion QV Nor is likely not overfilling its Roche lobe (Reynolds et al. 1992), so it is unlikely that the accreted material comes in with sufficient angular momentum to form a persistent disk, although there may be transient disk formation. However, to our knowledge, no searches for features that might indicate the existence of a transient disk (i.e., a $\sim$ few $\mathrm{mHz}$ quasi-periodic oscillation) have been performed.

The wind-accreting nature of the source, while it can explain the short-timescale variations in the pulse period, makes it more difficult to explain the long-term spin-up or spin-down trends observed. The torque reversal in particular is interesting: torque reversals observed in other sources have typically included large shifts in luminosity and/or dramatic spectral shifts (see, e.g., 4U 1626-67; Camero-Arranz et al. 2010). In 4U 1538-522, we see no shift in the X-ray luminosity across the reversal and the spectral parameters generally do not exhibit significant changes. Similar to 4U 1626-67 (Camero-Arranz et al. 2010) and 4U 1907+09 (Inam et al. 2009), the spin-up and spin-down rates on either side of the reversal are similar: $1.4 \times 10^{-14} \mathrm{~Hz} \mathrm{~s}^{-1}$ for the current spin-down trend, compared with a spin-up of $1.8 \times 10^{-14} \mathrm{~Hz} \mathrm{~s}^{-1}$ between 1990 and 2009. In the case of $4 \mathrm{U}$ $1907+09$ 's most recent torque reversal, Inam et al. suggested that the model of Perna et al. (2006) may be useful as they are able to produce torque reversals without large changes in luminosity or $|\dot{v}|$. However, this generally requires an Alfvén radius comparable to the corotation radius of the neutron star to produce the localized propeller effect on which the Perna et al. model relies. 4U 1538-522's slow rotation speed means its $\sim 10^{8}$ m corotation radius is $1-2$ orders of magnitude larger than its Alfvén radius, which is $\sim 4 \times 10^{6} \mathrm{~m}$ if spherical accretion is assumed (Lamb et al. 1973), so the recycling mechanism of the Perna et al. model is unlikely to be in effect. Meanwhile, Rubin et al. (1997) modeled 4U 1538-522's spin-up trend from 1991-1995 as simply the consequence of a random walk in the pulse frequency derivative (stemming from a random walk in the accretion torque on the neutron star) and proposed that this alone could be the source of the 1990 reversal, which would allow for the constancy of the system across the reversals. However, the nearly $20 \mathrm{yr}$ spin-up trend clearly indicates that the system is not solely random walking - while for Rubin et al. the expectation value for the frequency shift was within a factor of three of the observed shift, the most recent pulse period measurements are a factor of $\sim 8$ larger than the expected rms excursion. Without observations tracking the actual torque reversals (as was the case with $4 \mathrm{U} 1907+09)$, it is difficult to address the torque reversal in any more depth. 


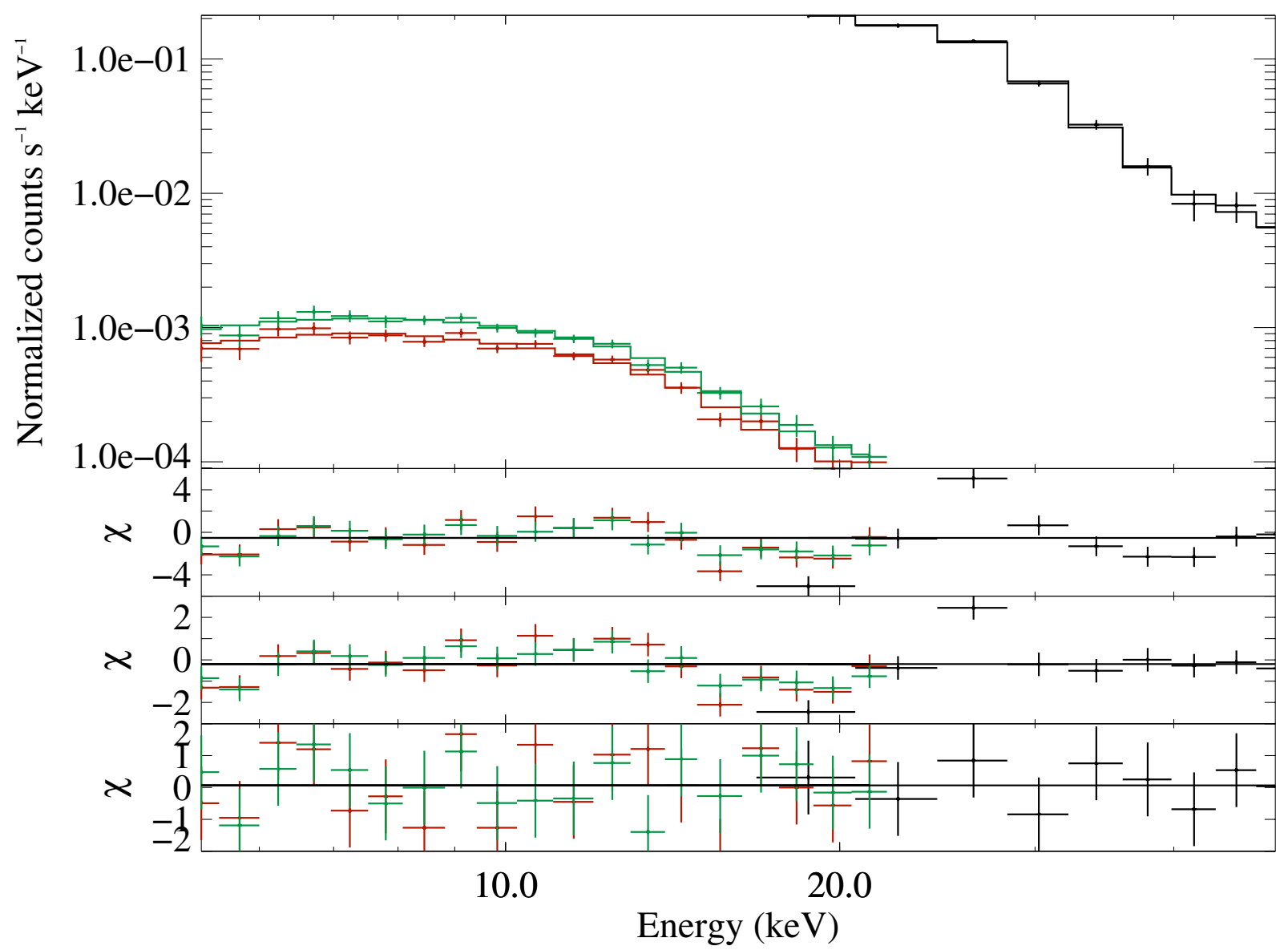

Figure 5. Spectrum of 4 U $1907+09$ with the best-fitting model, a PLCUT continuum modified by two GAUABS absorption features at 18.5 and 38 keV. The second panel displays the residuals from a pure PLCUT model, the third after only the harmonic CRSF has been added, and the lower panel displays the residuals with both CRSFs.

(A color version of this figure is available in the online journal.)

\subsection{Cyclotron Line Variability}

We detect absorption-like features, modeled as lines with Gaussian optical depth profiles, at $\sim 22$ and $\sim 50 \mathrm{keV}$ in $4 \mathrm{U}$ $1538-522$ and $\sim 18$ and $\sim 38 \mathrm{keV}$ in $4 \mathrm{U} 1907+09$, which we identify as CRSFs. These features are produced by the quantization of cyclotron motion in the strong magnetic field of the neutron star-photons with the appropriate energy and momentum to excite an electron into a new Landau level will see an increased scattering cross section. This manifests as a set of absorption-like features produced when photons are scattered out of the line of sight. For magnetic fields of strength $\lesssim 4 \times 10^{13} \mathrm{G}$, the magnetic field strength can be approximated from the energy of the fundamental cyclotron line via the "12-B-12" rule: $E_{\mathrm{cyc}}=(11.57 \mathrm{keV}) /(1+z) \times B_{12}$, where $z$ is the gravitational redshift in the scattering region and $B_{12}$ is the magnetic field strength in units of $10^{12} \mathrm{G}$. If we assume our measured CRSF energies reflect the magnetic field at the stellar surface, this implies a magnetic field strength of $\sim 2.1-2.3 \times 10^{12} \mathrm{G}$ for $4 \mathrm{U} 1538-522$ and $2.0 \times 10^{12} \mathrm{G}$ for $4 \mathrm{U} 1907+09$. Here, we have used masses for $4 \mathrm{U} 1538-522$ of $0.87 \pm 0.07$ and $1.104 \pm 0.177 M_{\odot}$ as determined by Rawls et al. (2011) and we have assumed a mass of $1.4 M_{\odot}$ for $4 \mathrm{U}$ $1907+09$. We have assumed a radius of $10 \mathrm{~km}$ for both sources.

A more in-depth investigation of the cyclotron line scattering region has been carried out by Becker et al. (2012), who predict the behavior of the CRSF in response to the luminosity of the source. In the supercritical regime, above the effective Eddington luminosity of the accretion column, they demonstrate that the height of the scattering region should scale with luminosity, producing a negative correlation between CRSF energy and luminosity, as the scattering region moves up into regions of weaker magnetic field. Meanwhile, in subcritical sources, the infalling material comes to a halt via a combination of gas and radiation pressure. An increase in luminosity means less gas pressure is necessary, lowering the scattering region and producing a positive $E_{\text {cyc }}-$ luminosity correlation. At even lower luminosity, below the Coulomb stopping limit $L_{\text {coul }}$ defined by Becker et al., the sum total gas and radiation pressure does not halt the infalling material before it impacts the star and the scattering should happen at or near the stellar surface. Here, the predicted behavior of the cyclotron line energy is somewhat uncertain, although Becker et al. suggest that there should not be any large correlation of $E_{\mathrm{cyc}}$ with luminosity.

Adopting distances of $6.4 \mathrm{kpc}$ for $4 \mathrm{U}$ 1538-522 (Reynolds et al. 1992) and $5 \mathrm{kpc}$ for $4 \mathrm{U} 1907+09$ (Cox et al. 2005) and determining the luminosity based on the $5-100 \mathrm{keV}$ flux, we calculated $L_{\text {crit }}$ and $L_{\text {coul }}$ using Becker et al.'s formulae and found that both sources lie firmly in the subcritical $\left(L<L_{\text {crit }}\right)$ regime, around or below $L_{\text {coul }}$. The exact location of $4 \mathrm{U}$ 1538-522 relative to $L_{\text {coul }}$ varies depending on which mass is used: with the very low-mass elliptical result, the measured 

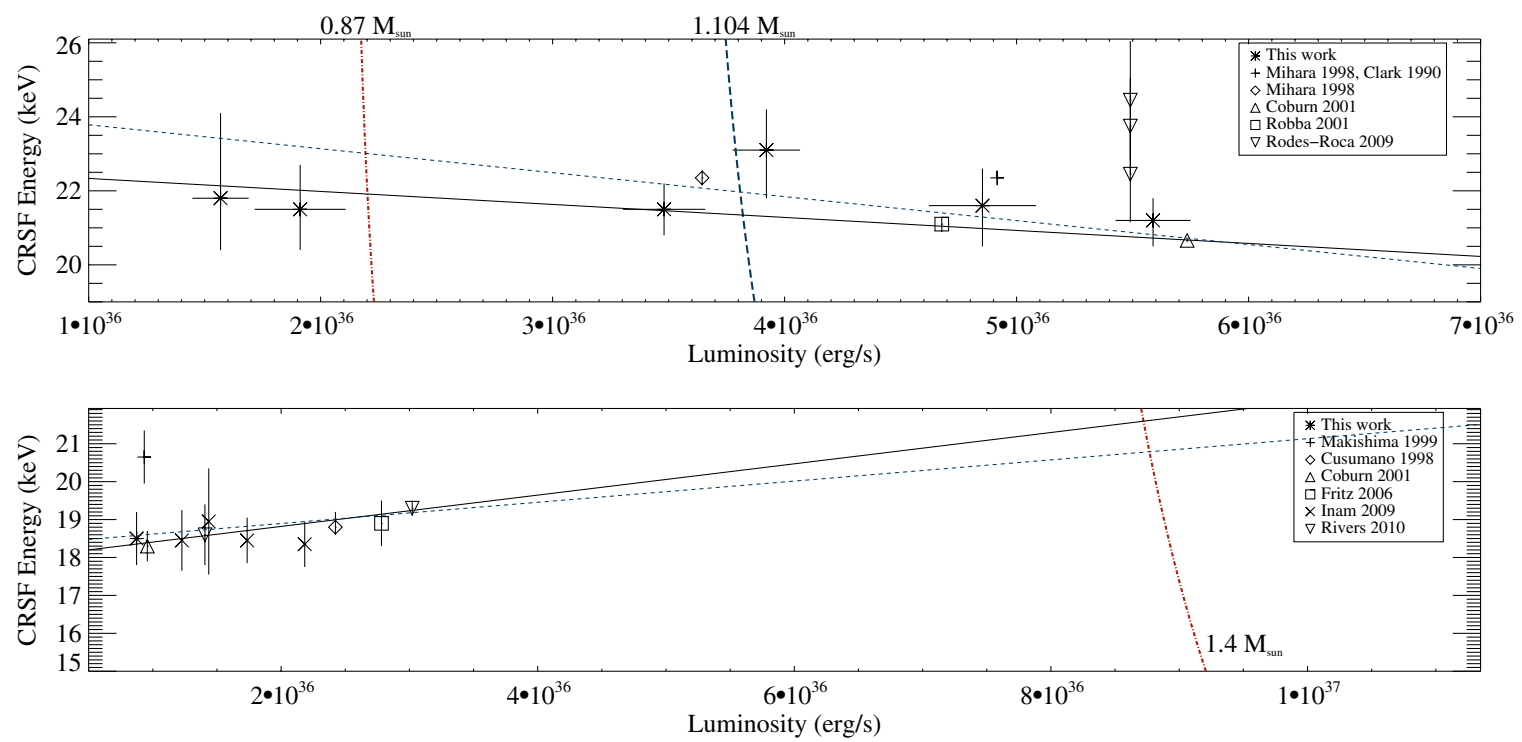

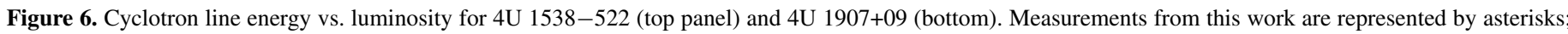

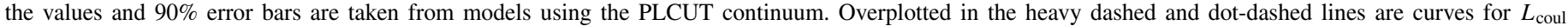

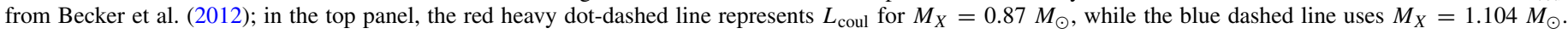

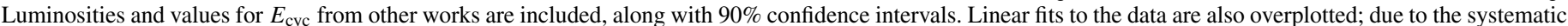

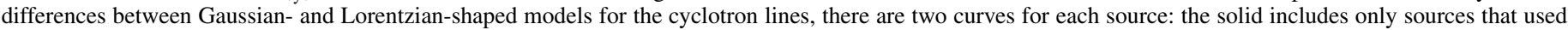

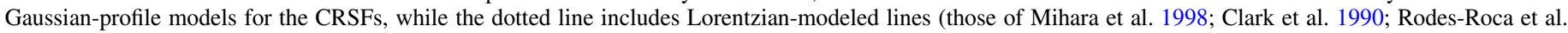
2009; Makishima et al. 1999; and Inam et al. 2009) shifted upward by $1.75 \mathrm{keV}$.

(A color version of this figure is available in the online journal.)

luminosities for the source lie mostly above the $L_{\text {coul }}$ cutoff, while the higher mass circular solution has the source straddling the $L_{\text {coul }}$ line. With no published mass measurements for $4 \mathrm{U}$ $1907+09$, we assume the canonical neutron star parameters of $M_{\mathrm{X}}=1.4 M_{\odot}$ and $R_{\mathrm{X}}=10 \mathrm{~km}$, which places the source far below the $L_{\text {coul }}$ cutoff. While the value for $L_{\text {coul }}$ can vary widely with mass $\left(L_{\text {coul }} \propto M_{\mathrm{X}}^{11 / 8}\right)$, unless $4 \mathrm{U} 1907+09$ has a very low mass similar to $4 \mathrm{U} 1538-522$, it is unlikely for it to move above $L_{\text {coul }}$ with an updated mass value. Our values for CRSF versus luminosity are plotted against $L_{\text {coul }}$ for both sources in Figure 6. To supplement this work's results, we also include the historical measurements of the energy of the primary CRSF for each source, limited to cases where the luminosity or flux was provided or could be derived from models. Based on our measured luminosity errors, we have assumed an error of $3 \%$ for these luminosity values.

Of course, when comparing results from different references, the issue of model choice must be addressed. Several analyses of 4U 1538-522 and 4U 1907+09 used a combination of the NPEX continuum and pseudo-Lorentzian line profiles for the CRSFs. This choice of models results in overall lower measured energies for the cyclotron lines and larger line widths compared with work that used Gaussians or the GAUABS model we have used. A formal treatment of this difference would be beyond the scope of this work: Mihara (1995) provides the analytical expression for the energy of the maximum of the pseudo-Lorentzian model, which one would expect to be close to the measured energy using a symmetric line shape, but the quantity is width-dependent $\left(E_{\max }=E+W^{2} / E\right)$ and Müller et al. (2013) point out that the width of the line can vary with the choice of the continuum model (e.g., for 4U 1907+09, Makishima et al. (1999), using NPEX, find a width of $\sim 7 \mathrm{keV}$, while Inam et al. (2009) find widths of $\sim 1-3 \mathrm{keV}$ when they use PLCUT). With this being the case, we opted to simply fit our data with a pseudo-Lorentzian (the CYCLABS model in XSPEC), obtaining values for $E_{\mathrm{c} 1}$ that were, on average, $1.75 \mathrm{keV}$ lower than those we found using GAUABS. This is roughly what one would expect using Mihara (1995)'s expression with a width of $\sim 6 \mathrm{keV}$.

To investigate the correlation between luminosity and CRSF energy, we performed linear fits and calculated 90\% error bars for the available references for each source; however, due to the systematic uncertainty introduced by correcting for the Lorentzian-modeled points, we defined two datasets for each source: the first only taking into account those results that used Gaussian line profiles similar to this work and the second with the "corrected" Lorentzian-modeled values as well as the Gaussian-modeled lines. The historical data for each source are insufficient to draw any conclusions based on the Lorentzianmodeled data alone. For 4U 1538-522, the Gaussian-modeled data amount to 8 points: the six values from Table 5 , along with measurements by Robba et al. (2001) and Coburn (2001). A linear fit to these data finds a slope of $-3 \pm 18 \mathrm{keV} /\left(10^{37} \mathrm{erg} \mathrm{s}^{-1}\right)$, consistent with zero. Pearson's $r$ for this dataset is -0.4 ; with six dof; this is consistent with no correlation. If we attempt to include the Lorentzian-modeled points in addition to the Gaussian-modeled points, the size of the applied correction becomes a deciding factor: any correction larger than $\sim 1.3 \mathrm{keV}$ results in a negative slope inconsistent with zero, with our $+1.75 \mathrm{keV}$ shift resulting in a slope of $-7 \pm 4 \mathrm{keV} /\left(10^{37} \mathrm{erg} \mathrm{s}^{-1}\right)$. Pearson's $r$ with this correction applied is 0.13 , still suggesting no correlation even with the additional data, although this is tempered by the large error bars on several of the data points. For 4U 1907+09, the Gaussian-modeled dataset consists of this work along with the results of Cusumano et al. (1998), Coburn (2001), Fritz et al. (2006), and Rivers et al. (2010). A linear fit to these data produces a positive slope of $4 \pm 3 \mathrm{keV} /\left(10^{37} \mathrm{erg} \mathrm{s}^{-1}\right)$ and a Pearson's $r$ of 0.92 , indicative of a correlation at the $p=0.02$ level. However, we should note that this positive slope is dependent to some extent on the measurements of Rivers et al. (2010) — specifically, the higher luminosity measurement of the 
CRSF. When that point is excluded from the dataset, Pearson's $r$ is still 0.93 , but the slope is no longer significant at the $90 \%$ level, returning a value of $3_{-6}^{+5} \mathrm{keV} /\left(10^{37} \mathrm{erg} \mathrm{s}^{-1}\right)$. This weakens somewhat any conclusion that 4U 1907+09 definitely has any CRSF-luminosity correlation. The discrepancy in the effect of correcting the Lorentzian-modeled results of Makishima et al. (1999) and Inam et al. (2009) makes the inclusion of these points more suspect than was the case for $4 \mathrm{U}$ 1538-522. Nonetheless, including the Lorentzian-modeled points, regardless of whether they are "corrected" upward or not, often still results in a positive slope. Only when the shift is greater than $\sim 1.8 \mathrm{keV}$, or the Rivers et al. (2010) point is excluded, does the slope become consistent with zero.

Considering the possibility of a correlation between CRSF energy and luminosity in $4 \mathrm{U} 1907+09$, it should be noted that a CRSF energy-luminosity correlation at this low of a luminosity has not been seen until now. The only source of comparable luminosity to $4 \mathrm{U} 1538-522$ or $4 \mathrm{U} 1907+09$ with a well-studied luminosity versus cyclotron line energy relationship is A $0535+26$ (Caballero et al. 2007), which shows no long-term trend. A $0535+26$ 's positive trend is limited to the pulse-to-pulse analysis by Klochkov et al. (2011), which only covers higher luminosity observations where the source was above $L_{\text {coul }}{ }^{8}$ With 4U 1538-522 and 4U 1907+09 operating at $L \lesssim L_{\text {coul }}$, the potential presence of a correlation is thus rather interesting-while the theoretical predictions for this luminosity regime are still somewhat speculative, the general concept was that at low luminosities, the scattering region would be essentially at the surface of the star, and an increase in luminosity would either do nothing or lift the scattering region up off the surface of the star, producing a negative trend in luminosity versus cyclotron line energy. We see something like this in $4 \mathrm{U} 1538-522$, which potentially has a negative slope in L-versus-E space. However, 4U 1907+09 shows evidence for a positive correlation. This would make 4U 1907+09 the fourth CRSF source to display a positive correlation with luminosity, after Hercules X-1 (Staubert et al. 2007), A 0535+26 (Klochkov et al. 2011), and GX 304-1 (Yamamoto et al. 2011). Hercules X-1 and GX 301-4 have L-versus-E slopes of similar magnitude to $4 \mathrm{U} 1907+09$, but they operate in a distinctly different luminosity regime, at $L_{\text {coul }}<L \lesssim L_{\text {crit }}$. The existence of a correlation in 4U 1907+09 would make the source somewhat of an outlier, with its luminosity sitting as far below $L_{\text {coul }}$ and $L_{\text {crit }}$ as it is. However, the weakness of the correlation and the low luminosity of the source make a conclusive determination difficult with the quality of data available.

\section{SUMMARY}

We have presented a spectral and timing analysis of the public INTEGRAL data for the HMXBs 4U 1538-522 and 4U $1907+09$. Our pulse period measurements for 4U 1538-522 support the Fermi GBM pulsar monitoring project's observation of a switch to a spin-down trend starting sometime in 2009, with our measured pulse periods agreeing strongly with their results. The source shows no significant spectral changes between its spin-up and spin-down epochs and its spectrum does not change much between higher and lower luminosities. We update the spectral results for $4 \mathrm{U} 1907+09$ to include the latest public

\footnotetext{
8 The astute reader may note that Reig \& Nespoli (2013) also saw evidence for a positive trend in $R X T E$ /Proportional Counter Array (PCA) data from A 0535+26's 2009 giant outburst. Nespoli et al. (2013), however, note that this trend was likely in part due to an oversubtraction of the PCA background, reducing the significance of the Reig \& Nespoli results.
}

INTEGRAL data; the results agree with much of the recent work on the source. In both sources, we detect two absorption features, identified as CRSFs. The fundamental CRSF in each source is detected very significantly at $\sim 22$ and $\sim 19 \mathrm{keV}$ in 4U 1538-522 and 4U 1907+09, respectively, with harmonics detected in $4 \mathrm{U} 1538-522$ at $\sim 50 \mathrm{keV}$ and in $4 \mathrm{U} 1907+09$ at $\sim 38 \mathrm{keV}$. An examination of our results along with those of past work reveals a possible negative correlation between CRSF energy and luminosity in $4 \mathrm{U} 1538-522$, while $4 \mathrm{U}$ 1907+09's CRSF energy-luminosity relationship has evidence for a positive slope, making it the fourth cyclotron line source to display this relationship.

P.B.H. acknowledges support from the National Aeronautics and Space Administration grant NNX09AT26G. I.C. acknowledges financial support from the French Space Agency CNES though CNRS. This work is based on observations made by the INTEGRAL satellite, a European Space Agency (ESA) project funded by ESA member states. We also thank the Fermi GBM Pulsar Project for their extended monitoring of multiple pulsars.

\section{REFERENCES}

Arnaud, K. A. 1996, in ASP Conf. Ser. 101, Astronomical Data Analysis Software and Systems V, ed. G. H. Jacoby \& J. Barnes (San Francisco, CA: ASP), 17

Baykal, A., İnam, S. c., \& Beklen, E. 2006, A\&A, 453, 1037

Becker, P. A., Klochkov, D., Schönherr, G., et al. 2012, A\&A, 544, A123

Becker, P. A., \& Wolff, M. T. 2007, ApJ, 654, 435

Becker, R. H., Swank, J. H., Boldt, E. A., et al. 1977, ApJL, 216, L11

Caballero, I., Kretschmar, P., Santangelo, A., et al. 2007, A\&A, 465, L21

Camero-Arranz, A., Finger, M. H., Ikhsanov, N. R., Wilson-Hodge, C. A., \& Beklen, E. 2010, ApJ, 708, 1500

Chernyakova, M., Neronov, A., Walter, R., \& Courvoisier, T. 2010, IBIS Analysis User Manual (Versoix: INTEGRAL Science Data Centre)

Clark, G. W. 2000, ApJL, 542, L131

Clark, G. W. 2004, ApJ, 610, 956

Clark, G. W., Woo, J. W., Nagase, F., Makishima, K., \& Sakao, T. 1990, ApJ, 353,274

Coburn, W. 2001, PhD thesis, Univ. California, San Diego

Corbet, R. H. D., Woo, J. W., \& Nagase, F. 1993, A\&A, 276, 52

Cox, N. L. J., Kaper, L., \& Mokiem, M. R. 2005, A\&A, 436, 661

Crampton, D., Hutchings, J. B., \& Cowley, A. P. 1978, ApJL, 225, L63

Cusumano, G., di Salvo, T., Burderi, L., et al. 1998, A\&A, 338, L79

Davison, P. J. N. 1977, MNRAS, 179, 35P

Davison, P. J. N., Watson, M. G., \& Pye, J. P. 1977, MNRAS, 181, 73P

Finger, M. H., Beklen, E., Narayana Bhat, P., et al. 2009, in 2009 Fermi Symp., ed. W. N. Johnson \& D. J. Thompson (Washington, DC: eConf. Proc.), arXiv:0912.3847

Fritz, S., Kreykenbohm, I., Wilms, J., et al. 2006, A\&A, 458, 885

Giacconi, R., Kellogg, E., Gorenstein, P., Gursky, H., \& Tananbaum, H. 1971, ApJL, 165, L27

Giacconi, R., Murray, S., Gursky, H., et al. 1974, ApJS, 27, 37

Ilovaisky, S. A., Chevalier, C., \& Motch, C. 1979, A\&A, 71, L17

Inam, S. Ç., Şahiner, Ş., \& Baykal, A. 2009, MNRAS, 395, 1015

in 't Zand, J. J. M., Baykal, A., \& Strohmayer, T. E. 1998, ApJ, 496, 386

Klein, R. I., Arons, J., Jernigan, G., \& Hsu, J. J.-L. 1996, ApJL, 457, L85

Klochkov, D., Staubert, R., Santangelo, A., Rothschild, R. E., \& Ferrigno, C. 2011, A\&A, 532, A126

Kostka, M., \& Leahy, D. A. 2010, MNRAS, 407, 1182

Lamb, F. K., Pethick, C. J., \& Pines, D. 1973, ApJ, 184, 271

Larsson, S. 1996, A\&AS, 117, 197

Leahy, D. A., Darbro, W., Elsner, R. F., et al. 1983, ApJ, 266, 160

Lebrun, F., Leray, J. P., Lavocat, P., et al. 2003, A\&A, 411, L141

Lund, N., Budtz-Jørgensen, C., Westergaard, N. J., et al. 2003, A\&A, 411, L231

Makishima, K., Kawai, N., Koyama, K., et al. 1984, PASJ, 36, 679

Makishima, K., Koyama, K., Hayakawa, S., \& Nagase, F. 1987, ApJ, 314, 619

Makishima, K., Mihara, T., Nagase, F., \& Tanaka, Y. 1999, ApJ, 525, 978

Marshall, N., \& Ricketts, M. J. 1980, MNRAS, 193, 7P

Meszaros, P., \& Nagel, W. 1985, ApJ, 299, 138

Mihara, T. 1995, PhD thesis, Dept. Physics, Univ. Tokyo

Mihara, T., Makishima, K., \& Nagase, F. 1998, AdSpR, 22, 987 
Mukherjee, U., Raichur, H., Paul, B., Naik, S., \& Bhatt, N. 2006, JApA, 27, 411

Müller, S., Ferrigno, C., Kühnel, M., et al. 2013, A\&A, 551, A6

Müller, S., Kühnel, M., Caballero, I., et al. 2012, A\&A, 546, A125

Nespoli, E., Fabregat, J., \& Mennickent, R. E. 2008, A\&A, 486, 911

Nespoli, E., Klochkov, D., Caballero, I., Reig, P., \& Kretschmar, P. 2013, in Spectral/Timing Properties of Accreting Objects: From X-Ray Binaries to AGN (Madrid: ESA/ESAC)

Perna, R., Bozzo, E., \& Stella, L. 2006, ApJ, 639, 363

Rawls, M. L., Orosz, J. A., McClintock, J. E., et al. 2011, ApJ, 730, 25

Reig, P., \& Nespoli, E. 2013, A\&A, 551, A1

Reynolds, A. P., Bell, S. A., \& Hilditch, R. W. 1992, MNRAS, 256, 631

Rivers, E., Markowitz, A., Pottschmidt, K., et al. 2010, ApJ, 709, 179

Robba, N. R., Burderi, L., Di Salvo, T., Iaria, R., \& Cusumano, G. 2001, ApJ, 562,950

Rodes-Roca, J. J., Page, K. L., Torrejón, J. M., Osborne, J. P., \& Bernabéu, G. 2010, A\&A, 526, A64
Rodes-Roca, J. J., Torrejón, J. M., Kreykenbohm, I., et al. 2009, A\&A, 508, 395 Rubin, B. C., Finger, M. H., Scott, D. M., \& Wilson, R. B. 1997, ApJ, 488, 413 Şahiner, Ş., Inam, S. Ç., \& Baykal, A. 2011, in AIP Conf. Ser. 1379, Astrophysics of Neutron Stars 2010, ed. E. Göğüş, T. Belloni, \& Ü. Ertan (Melville, NY: AIP), 214

Şahiner, Ş., Inam, S. Ç., \& Baykal, A. 2012, MNRAS, 421, 2079

Schönherr, G., Wilms, J., Kretschmar, P., et al. 2007, A\&A, 472, 353

Schwarm, F. 2013, PhD thesis, Univ. Erlangen-Nuremberg

Schwarm, F.-W., Schönherr, G., Becker, P. A., et al. 2013, AAS/High Energy Astrophysics Division, Vol. 13 (Monterey, CA: AAS), 126.31

Staubert, R., Shakura, N. I., Postnov, K., et al. 2007, A\&A, 465, L25

Ubertini, P., Lebrun, F., Di Cocco, G., et al. 2003, A\&A, 411, L131

van Kerkwijk, M. H., van Oijen, J. G. J., \& van den Heuvel, E. P. J. 1989, A\&A, 209,173

van Kerkwijk, M. H., van Paradijs, J., \& Zuiderwijk, E. J. 1995, A\&A, 303, 497

Yamamoto, T., Sugizaki, M., Mihara, T., et al. 2011, PASJ, 63, 751 